\title{
ARTICLE OPEN \\ Randomized phase-II evaluation of letrozole plus dasatinib in hormone receptor positive metastatic breast cancer patients
}

Devchand Paul $^{1,2}$, Svetislava J. Vukelja ${ }^{1,3}$, Frankie Ann Holmes $\mathbb{D}^{1,4}$, Joanne L. Blum ${ }^{1,5}$, Kristi J. Mclntyre ${ }^{1,6}$, Deborah L. Lindquist ${ }^{1,7}$, Cynthia R. Osborne ${ }^{1,5}$, Ines J. Sanchez ${ }^{1,8}$, Jerome H. Goldschmidt ${ }^{1,9}$, Yunfei Wang ${ }^{1}$, Lina Asmar ${ }^{1}$, Lewis Strauss ${ }^{10}$ and Joyce O'Shaughnessy ${ }^{1,5 *}$

The non-receptor tyrosine kinase Src activation plays a role in the malignant progression of breast cancer, including development of endocrine therapy resistance and survival of bone metastases. This study investigated whether adding Src kinase inhibitor dasatinib to aromatase inhibitor (Al) therapy improved outcomes in estrogen receptor (ER)-positive, HER2-negative metastatic breast cancer (MBC). Postmenopausal patients with ER-positive, HER2-negative MBC (0-1 prior chemotherapies and no prior AI for $\mathrm{MBC}$ ) were eligible for this non-comparative, parallel group, phase-ll study. Patients were randomized to letrozole (2.5 mg/day PO) alone or with dasatinib (100 mg/day PO). Patients with disease progression on letrozole alone could crossover to dasatinib plus continued letrozole. The primary endpoint was clinical-benefit-rate (CBR; complete response + partial response + stable disease $\geq 6$ months). A total of 120 patients were randomized. The CBR of $71 \%$ (95\% Cl 58-83\%) was observed with letrozole + dasatinib versus the projected CBR of the combination of $56 \%$. The CBR of $66 \%$ (95\% Cl 52-77\%) with letrozole alone also exceeded the projected CBR of $39 \%$ with letrozole alone. The CBR was $23 \%$ in the crossover arm of letrozole plus dasatinib in patients progressing on letrozole alone. Median progression-free survival with the combination was 20.1 months and 9.9 months with letrozole alone. Letrozole plus dasatinib was well tolerated, although $26 \%$ of patients required dasatinib dose reductions. In this non-comparative phase-II trial, the CBR of $71 \%$ and the median PFS of 20.1 months with letrozole + dasatinib are encouraging and suggest that dasatinib may inhibit the emergence of acquired resistance to Al therapy.

npj Breast Cancer (2019)5:36

; https://doi.org/10.1038/s41523-019-0132-8

\section{INTRODUCTION}

Src is a pleiotropic, membrane-associated, non-receptor tyrosine kinase that has been implicated in the proliferation, survival, migration, and invasion of breast cancer cells, as well as the development of resistance to therapy. ${ }^{1-3}$ In vitro studies have shown that Src interacts with a variety of signaling molecules including growth factor receptors, integrins, and steroid hormone receptors. ${ }^{1-3}$ In breast cancer cells, membrane-associated estrogen receptor-alpha (ERa) has been shown to complex with Src and phosphatidylinositol 3-kinase (PI3K) to drive growth and endocrine therapy resistance. ${ }^{4-6}$ Levels of ERa and Src are inversely correlated in primary breast cancers, and Src has been shown to increase the proteolytic degradation of Era. ${ }^{7}$ Combining Src inhibitors with endocrine agents can reduce the emergence of acquired endocrine resistance in preclinical studies. ${ }^{1,8}$ Further, in a phase-II clinical study of the Src inhibitor bosutinib as monotherapy in 73 patients with previously treated advanced or metastatic breast cancer (MBC), all four responses occurred in patients with hormone receptor (HR)-positive disease. ${ }^{9}$ Finally, Src is involved in the regulation of osteoclastmediated bone turnover, ${ }^{10}$ and has been implicated in the survival and outgrowth of breast cancer cells in the bone marrow microenvironment. ${ }^{2}$

Dasatinib is a potent oral tyrosine kinase inhibitor (TKI) with specificity for a number of related kinases including BCR-ABL, SrC, $\mathrm{c}-\mathrm{KIT}$, platelet-derived growth factor receptor, and TEC family kinases. ${ }^{11}$ Dasatinib has been approved for the treatment of newly diagnosed Philadelphia chromosome-positive $(\mathrm{Ph}+)$ chronic myelogenous leukemia $(\mathrm{CML})$ in chronic phase, as well as $\mathrm{Ph}+$ CML or acute lymphoblastic leukemia (ALL) resistant to or intolerant of prior imatinib. ${ }^{11}$ In preclinical models of tamoxifenresistant breast cancer, dasatinib alone or in combination with tamoxifen or letrozole inhibited tumor growth. ${ }^{3,5}$ In a phase-II clinical trial, dasatinib monotherapy (100 mg or $70 \mathrm{mg}$ BID) produced 2 confirmed partial responses (PRs) and 6 disease stabilizations $\geq 16$ weeks in 45 evaluable patients with advanced, pretreated HR-positive breast cancer. ${ }^{12}$ Adverse events were similar to those observed in $\mathrm{CML}$, including fatigue, diarrhea, headache, nausea, and pleural effusion. Dasatinib has also demonstrated bone anabolic and anti-resorptive effects in preclinical systems. ${ }^{13,14}$

This randomized non-comparative phase-II study with a concurrent control arm was designed to evaluate the efficacy and safety of dasatinib plus letrozole for postmenopausal patients with ER-positive, HER2-negative $\mathrm{MBC}$, and to determine if this combination could delay the development of endocrine therapy resistance. In addition, correlative exploratory analyses of protein expression by reverse phase protein array (RPPA) and activation patterns were performed to identify potential biomarkers associated with sensitivity or resistance to letrozole or letrozole plus dasatinib.

${ }^{1}$ US Oncology Research, Inc., 10101 Woodloch Forest Dr., The Woodlands, TX 77380, USA. ${ }^{2}$ Rocky Mountain Cancer Centers, 4700 East Hale Park Way \#400, Denver, CO 80220, USA. ${ }^{3}$ Texas Oncology-Tyler, 910 E Houston St \#100, Tyler, TX 75702, USA. ${ }^{4}$ Texas Oncology-Houston Memorial City, 925 Gessner \#550, Houston, TX 77024, USA. ${ }^{5}$ Texas Oncology at Baylor University Medical Center, 3410 Worth Street, Dallas, TX 75246, USA. ${ }^{6}$ Texas Oncology-Dallas Presbyterian Hospital, 8196 Walnut Hill \#100, Dallas, TX 75231, USA. ${ }^{7}$ Arizona Oncology Associates, 3700W State Route 89A, Sedona, AZ 86336, USA. ${ }^{8}$ Texas Oncology, 1901 Grandview, El Paso, TX 79902 , USA. ${ }^{9}$ Blue Ridge Cancer Care, 2600 Research Center Drive, Suite A, Blacksburg, VA 24060, USA. ${ }^{10}$ Bristol-Myers Squibb, 5 Research Pkwy, Wallingford, CT 06492, USA. *email: Joyce.Oshaughnessy@usoncology.com 


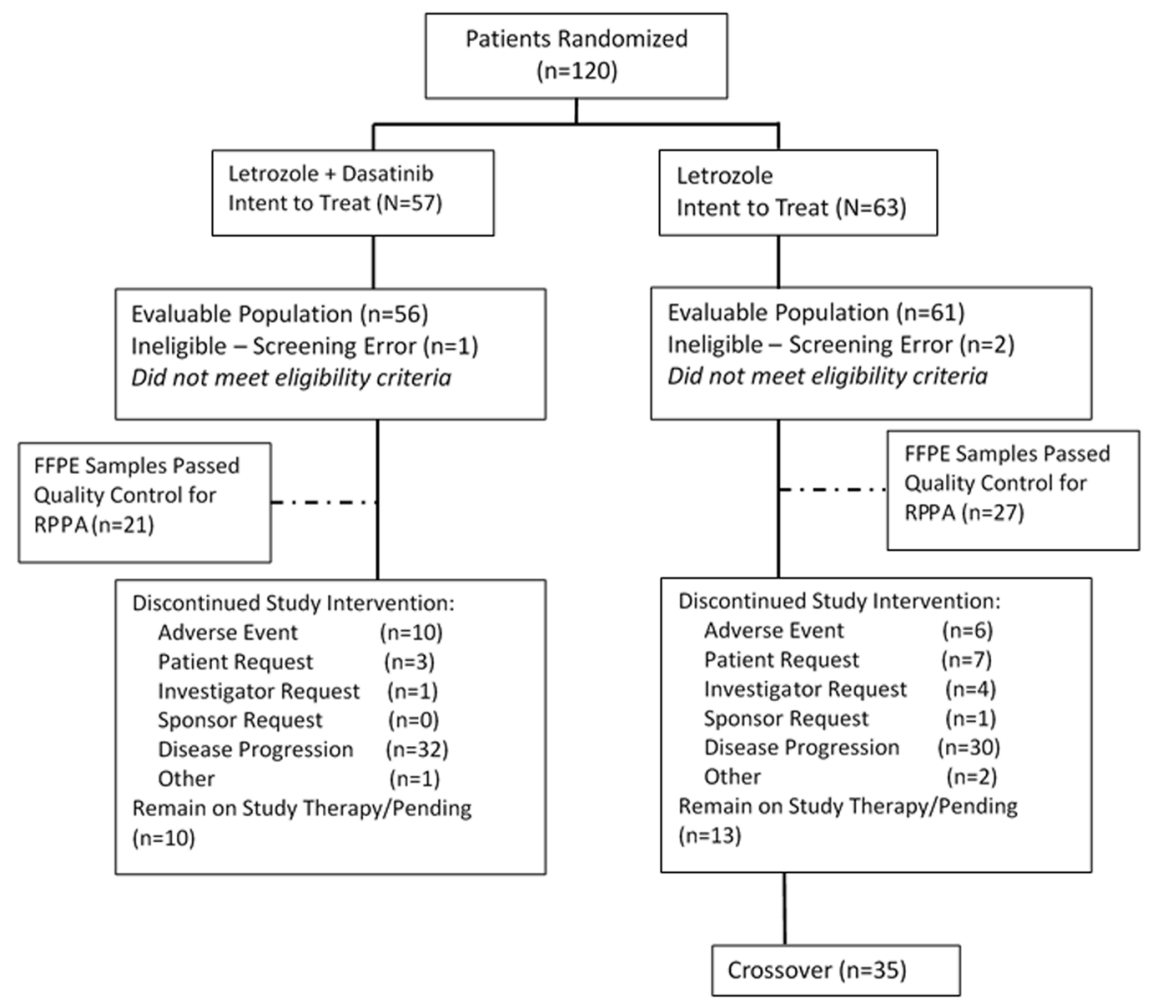

Fig. 1 CONSORT diagram. Treatment assignment

\section{RESULTS}

Patient characteristics

A total of 120 patients were enrolled and randomized, 57 to letrozole plus dasatinib and 63 to letrozole monotherapy between 2008 and 2013. (CONSORT diagram-Fig. 1) Patient characteristics were generally well balanced between arms, except that there were more de novo metastatic and endocrine naïve patients in the combination arm (Table 1). Approximately half of patients had received prior chemotherapy, either in the adjuvant or metastatic settings. Approximately half of the patients who had developed recurrent disease had a DFI of $\leq 2$ years.

\section{Safety and tolerability}

Combination treatment with letrozole plus dasatinib was generally well tolerated, and the majority of adverse events were grade 1/2 (Table 2). The most frequent AEs (all grades) were fatigue (42.1\%), nausea $(38.6 \%)$, rash $(26.3 \%)$, anemia $(24.6 \%)$, and neutropenia (22.8\%). Grade 3 AEs were uncommon, and included rash and edema ( 2 events each), and fatigue, anemia, neutropenia, arthralgia, and pleural effusion (1 event each). One patient in the combination arm experienced two grade $4 \mathrm{AEs}$ considered related to study drug: respiratory failure and acute renal failure; both events resolved. Pleural effusion occurred in 9 patients (16\%), and all but one were grade 1 or 2 events. No grade $3 / 4$ liver dysfunction was observed. Fifteen patients $(26 \%)$ in the letrozole plus dasatinib arm required a dasatinib dose reduction. A total of 10 patients (17.5\%) in the combination arm and 6 patients $(9.5 \%)$ in the letrozole alone arm discontinued study treatment due to an AE. Four patients remained on dasatinib plus letrozole for $3+$ years.

\section{Efficacy}

The primary endpoint of $C B R(C R+P R+S D \geq 6$ months) of this non-comparative trial was the $\mathrm{CBR}$ of combined letrozole plus dasatinib which was $71 \%(95 \% \mathrm{CI} 58-83 \%)$, versus the projected CBR of $56 \%$ with letrozole plus dasatinib. The CBR observed with letrozole alone was $66 \%$ (95\% Cl 52-77\%) which also exceeded the projected CBR of $39 \%$ with letrozole alone. The ORRs were $23 \%$ (95\% Cl 13-36\%) with letrozole plus dasatinib and $25 \%$ (95\% Cl 15-37\%) with letrozole alone in the response-evaluable population. In the subset of 35 patients who crossed over to dasatinib plus letrozole following disease progression on letrozole alone, the CBR was $23 \%$ (8/35), with a median PFS of 3.7 months.

Median PFS was 20.1 months with the letrozole/dasatinib combination and was 9.9 months with letrozole alone (Fig. 2). Median OS was 48.6 months with the combination and was 41.9 months with letrozole alone.

\section{Bone mineral density}

The percentage of patients with osteopenia, defined in this study as a T score $<-1.5$ in either the lumbar spine or femur, decreased in patients receiving dasatinib plus letrozole, from $32 \%$ at baseline to $14 \%$ on study therapy (obtained at least 6 months following initiation of study treatment). There was no change in BMD in patients treated with letrozole alone, with $38 \%$ of patients being classified as osteopenic at baseline, and $32 \%$ on study therapy. Bisphosphonates therapy was allowed after completion of the first 8 weeks of study treatment; 18 patients on the dasatinib plus letrozole arm received bisphosphonates while on study (1 oral, 17 intravenous), as did 23 patients on the letrozole monotherapy arm ( 2 oral, 21 intravenous). The duration of bisphosphonate use was not captured.

\section{Exploratory biomarker analysis}

Primary breast cancer FFPE tissues were available for 88 patients, and of these, 48 were adequate for RPPA. Median PFS rates for the 48 patients with adequate tissue for RPPA were 
Table 1. Demographics and baseline characteristics (ITT population)

\begin{tabular}{|c|c|c|c|}
\hline Parameter & & $\begin{array}{l}\text { Letrozole }+ \\
\text { Dasatinib } \\
(n=57(\%))\end{array}$ & $\begin{array}{l}\text { Letrozole } \\
(n=63(\%))\end{array}$ \\
\hline Age, years (range) & Median & $\begin{array}{l}62.3 \\
(37.4,80.9)\end{array}$ & $\begin{array}{l}61.3 \\
(35.6,86.9)\end{array}$ \\
\hline \multicolumn{4}{|c|}{ DFI in Recurrent Metastatic Patients } \\
\hline & $<=2$ years & $27(47)$ & $34(54)$ \\
\hline & $>2$ years & $30(53)$ & $29(46)$ \\
\hline & $\begin{array}{l}\text { Median (Range) } \\
\text { in months }\end{array}$ & $27.5(0,277)$ & $21.2(0,290)$ \\
\hline De Novo stage IV & & $24(42)$ & $20(32)$ \\
\hline \multirow{3}{*}{$\begin{array}{l}\text { ECOG } \\
\text { performance status }\end{array}$} & 0 & $33(58)$ & $40(64)$ \\
\hline & 1 & $24(42)$ & $22(35)$ \\
\hline & 2 & 0 & $1(2)$ \\
\hline \multirow[t]{5}{*}{ Histological grade } & G1 & $8(14)$ & $6(10)$ \\
\hline & G2 & $22(39)$ & $28(44)$ \\
\hline & G3 & $17(30)$ & $14(22)$ \\
\hline & $\begin{array}{l}\text { GX (Grade cannot } \\
\text { be assessed) }\end{array}$ & 0 & $5(8)$ \\
\hline & Unknown & $10(18)$ & $10(16)$ \\
\hline \multirow[t]{4}{*}{ Sites of metastasis } & Bone & $42(74)$ & $44(70)$ \\
\hline & $\begin{array}{l}\text { Lymph node/ } \\
\text { soft tissue }\end{array}$ & $18(32)$ & $18(29)$ \\
\hline & Visceral-liver & $10(18)$ & $12(19)$ \\
\hline & Visceral-lung & $8(14)$ & $10(16)$ \\
\hline \multirow[t]{3}{*}{$\begin{array}{l}\text { Assessment type } \\
\text { (evaluable population) }\end{array}$} & $\begin{array}{l}\text { Measurable } \\
\text { by RECIST }\end{array}$ & $34(60)$ & $46(73)$ \\
\hline & Not measurable & $22(39)$ & $15(24)$ \\
\hline & Missing & $1(2)$ & $2(3)$ \\
\hline \multirow[t]{5}{*}{ Prior chemotherapy } & Yes & $26(46)$ & $33(52)$ \\
\hline & Adjuvant & $22(39)$ & $30(48)$ \\
\hline & Metastatic & $6(11)$ & $3(5)$ \\
\hline & No & $31(54)$ & $29(46)$ \\
\hline & Unknown & 0 & $1(2)$ \\
\hline \multirow{7}{*}{$\begin{array}{l}\text { Prior endocrine } \\
\text { therapy }\end{array}$} & Yes & $22(39)$ & $32(51)$ \\
\hline & Adjuvant & $19(33)$ & $29(46)$ \\
\hline & $\begin{array}{l}\text { Adjuvant- } \\
\text { tamoxifen }\end{array}$ & $18(32)$ & $23(37)$ \\
\hline & $\begin{array}{l}\text { Adjuvant- } \\
\text { others } \\
\text { (aromatase } \\
\text { inhibitor) }\end{array}$ & $1(2)$ & $6(10)$ \\
\hline & Metastatic & $4(7)$ & $3(5)$ \\
\hline & $\begin{array}{l}\text { Metastatic- } \\
\text { tamoxifen }\end{array}$ & $3(5)$ & $2(3)$ \\
\hline & No & $35(61)$ & 31 (49) \\
\hline \multirow[t]{3}{*}{ ER|PR status } & $\mathrm{ER}+\mathrm{PR}+$ & $53(93)$ & $57(90)$ \\
\hline & $\mathrm{ER}+\mathrm{PR}-$ & $3(5)$ & $6(10)$ \\
\hline & ER-PR+ & $1(2)$ & 0 \\
\hline
\end{tabular}

very similar to that of the entire study population: 19.2 months for letrozole plus dasatinib $(n=21)$ and 9.2 months for letrozole alone $(n=27)$.

Approximately $90 \%$ of the primary breast cancers overexpressed HER3, p-mTOR, p-4EBP1, p-JAK2, p-STAT3, and 50\% overexpressed p-Paxillin. Having PFS $\leq 6$ months, i.e., no evidence of clinical benefit, was associated with HER3 $(p<0.01)$, p-HER3
( $p=0.03), \mathrm{p}$-Src $(p=0.06), \mathrm{p}$-Paxillin $(p=0.02)$, or $\mathrm{p}-\mathrm{MET}(p=0.05)$ expression in letrozole monotherapy-treated patients but expression of these phospho-proteins was not associated with poor outcome in the letrozole plus dasatinib-treated patients (Supplemental Table 2). However, in both arms, having PFS $\leq 6$ months was significantly associated with co-expression of p-HER3 and p-Src (Supplemental Tables 3 and 4). AR expression correlated with expression of $\mathrm{p}$-HER2, epidermal growth factor receptor (EGFR), p-EGFR, p-IGFR, p-ERK in letrozole plus dasatinib-treated patients who had PFS $\leq 6$ months but not in letrozole-treated patients who had PFS $\leq 6$ months.

\section{DISCUSSION}

This non-comparative phase-II trial in patients with HR-positive/ HER2-negative $M B C$ receiving their first $A I$ in the metastatic setting demonstrated the primary endpoint, CBR, of $71 \%$ with letrozole plus dasatinib versus the projected CBR of $56 \%$ with letrozole plus dasatinib. The CBR rate of $66 \%$ in the letrozolealone control arm also exceeded the assumed value of $39 \%$. The higher than expected CBR with letrozole alone observed in this trial compared with the original first-line letrozole trial may have been due in part to the higher proportion of de novo MBC patients enrolled in this trial (37\% vs $32 \%$ in Mouridsen et al). ${ }^{15}$ Recent phase-III trials combining first-line letrozole plus a CDK 4/ 6 inhibitor have shown CBRs of 70 to $73 \%$ with letrozole plus placebo, similar to the 66 and $71 \%$ CBRs observed with letrozole alone and with letrozole plus dasatinib in the current trial. ${ }^{16,17}$ Analysis of the secondary endpoint of PFS showed a longer than expected median PFS of 20.1 months with combined letrozole plus dasatinib. The median PFS of 9.9 months observed with letrozole alone is similar to the median PFS of 10 months reported with first-line letrozole in the Mouridsen pivotal trial. ${ }^{15}$ The long duration of PFS with letrozole/dasatinib could also have been seen because $42 \%$ of these patients had de novo metastatic disease and because $61 \%$ of patients were endocrine therapy-naïve.

The durable PFS observed with letrozole/dasatinib raises the hypothesis that dasatinib may slow the development of acquired resistance to letrozole. Results of two randomized phase-II studies which compared either exemestane or fulvestrant alone versus in combination with dasatinib showed no significant improvement in PFS with the addition of the Src inhibitor in patients with MBC that was resistant to a non-steroidal Al. ${ }^{18,19}$ It is possible that inhibiting Src can delay the remodeling of signaling pathways that lead to Al resistance in initially Alsensitive breast cancers by inhibiting the formation of the ER/ EGFR/Src complex. ${ }^{20}$ Also, in preclinical models, dasatinib increases ERa expression by inhibiting Src-mediated ERa degradation, and this may have contributed to the prolonged PFS observed with dasatinib plus letrozole. ${ }^{7}$

The $A E$ profile observed with letrozole plus dasatinib in this study is consistent with the safety record of dasatinib in $C M L$, as well as the AE results from the phase-II study in combination with exemestane. ${ }^{18}$ Overall, the combination was well tolerated, and although $26 \%$ of patients required dose reductions, the rate of grade 3/4 AEs, including pleural effusion, was low. No serious hepatotoxicity was observed, in contrast to results observed with another Src/Abl TKI, bosutinib. Two phase-II studies evaluated bosutinib in combination with first-line letrozole or second-line exemestane in postmenopausal patients with HR-positive/HER2negative $\mathrm{MBC} .^{21,22}$ Although these combinations demonstrated anti-tumor activity, both trials were terminated early due to unfavorable safety profiles, including a $26-38 \%$ incidence of hepatotoxicity, and with approximately two-thirds of patients experiencing diarrhea.

A secondary objective of this trial was to explore the impact of dasatinib on BMD assessed by DXA scan. In the dasatinib arm, 
Table 2. Adverse events occurring in $\geq 10 \%$ of patients on study treatment

\begin{tabular}{|c|c|c|c|c|c|c|c|c|}
\hline Edema & $1(2)$ & $4(7)$ & $2(4)$ & $7(12)$ & 0 & 0 & 0 & 0 \\
\hline Fatigue & $15(26)$ & $8(14)$ & $1(2)$ & $24(42)$ & $3(5)$ & $2(3)$ & 0 & $5(8)$ \\
\hline Arthralgia & $6(11)$ & $2(4)$ & $1(2)$ & $9(15)$ & $1(2)$ & $2(3)$ & 0 & $3(5)$ \\
\hline Pleural Effusion & $4(7)$ & $4(7)$ & $1(2)$ & $9(16)$ & 0 & 0 & 0 & 0 \\
\hline Hot Flashes & $7(12)$ & $1(2)$ & 0 & $8(14)$ & $10(16)$ & $2(3)$ & $1(2)$ & $13(20)$ \\
\hline Nausea & $15(26)$ & $7(12)$ & 0 & $22(39)$ & $5(8)$ & 0 & 0 & $5(8)$ \\
\hline Diarrhea & $7(12)$ & $3(5)$ & 0 & $10(18)$ & 0 & 0 & 0 & 0 \\
\hline
\end{tabular}

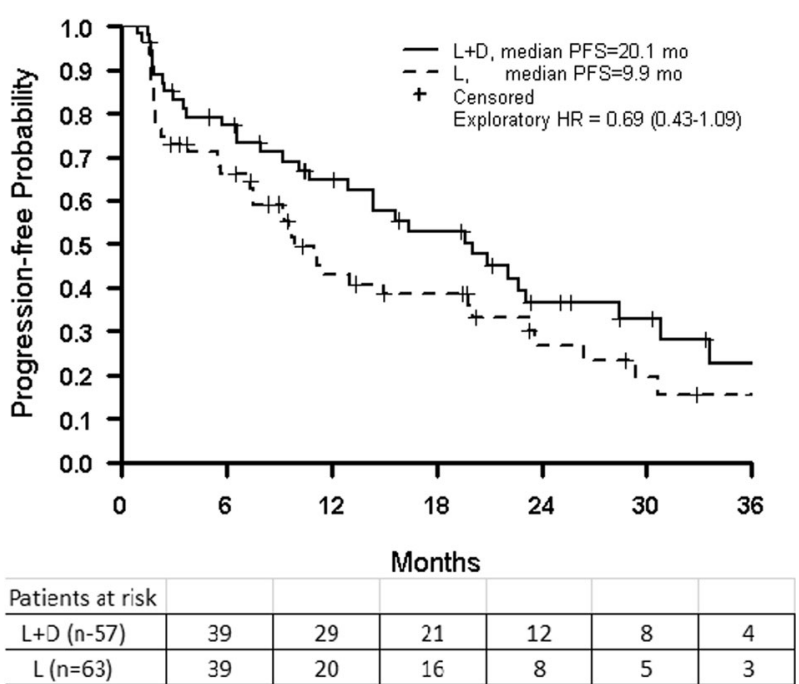

Fig. 2 Estimates of progression-free survival in the intent-to-treat population. $L$ letrozole; $L+D$ letrozole plus dasatinib

the proportion of patients with osteopenia in the lumbar spine and/or hip was reduced from $32 \%$ at baseline to $14 \%$ on study treatment (Supplemental Fig. 1). It is plausible that dasatinib could improve bone density as Src kinase activity is essential to osteoclast development and function, ${ }^{23,24}$ and clinically achievable levels of dasatinib inhibit osteoclast activity. ${ }^{13,14}$ In preclinical studies in mice, low-dose dasatinib has been shown to promote osteoblast differentiation and matrix mineralization, and at the same time reduce osteoclast formation, differentiation, and resorption. ${ }^{13}$ The finding that some patients may have had improvement in their BMD on dasatinib is preliminary as DXA scans were missing in some patients, calibration of DXA imaging across centers was not performed, nor was central review of the scans (Fig. 3).

Our exploratory biomarker analyses in patients' primary breast cancers failed to identify any strong predictors of benefit from dasatinib in the metastatic setting but did generate interesting hypotheses for future investigation. A limitation of these analyses

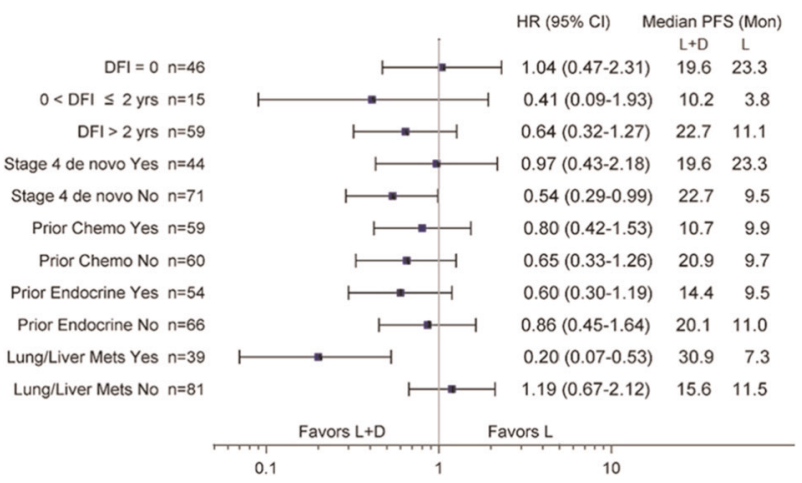

Fig. 3 Forest plot of hazard ratios for progression-free survival by population subgroups

is that some primary breast cancers may not harbor activated pathways that become manifest in established metastatic disease. Nonetheless, lack of clinical benefit with either letrozole or letrozole plus dasatinib (PFS $\leq 6$ months) was associated with coexpression of $\mathrm{p}$-Src and $\mathrm{p}$-HER3 (and with co-expressed $\mathrm{p}$-Src and EGFR in letrozole-treated patients) suggesting that coordinate activation of HER3 and Src may result in both letrozole and dasatinib resistance. The importance of HER3 signaling in endocrine resistance was recently demonstrated in a randomized phase-Il study which showed a significant improvement in PFS when the HER3-targeted monoclonal antibody MM-121 was added to exemestane in patients with HR-positive MBC with high heregulin expression and low HER2 expression. ${ }^{25}$

$A R$ expression in the primary breast cancers correlated with expression of p-HER2, EGFR, p-EGFR, p-IGFR, and p-ERK in patients who did not benefit from letrozole plus dasatinib (PFS $\leq 6$ months), suggesting a potential interaction between AR and HER family receptors in letrozole/dasatinib resistance. Gene expression profiling has shown elevated AR and reduced ERa expression in tamoxifen-resistant breast cancers, while overexpression of AR can induce tamoxifen resistance in preclinical breast cancer models. ${ }^{26}$ These findings are hypothesis-generating due to the multiplicity of comparisons in this small sample set. 
Moulder et al. utilized gene expression profiling on dasatinibsensitive and -resistant cell lines to develop three potential predictive signatures of dasatinib sensitivity or resistance: a dasatinib sensitivity index, a Src pathway activity index, and a dasatinib target index. ${ }^{27}$ Among 133 breast cancer samples, the signatures identified distinct subsets that were predicted to be sensitive to dasatinib. However, because the patients had not been treated with dasatinib, no clinical correlations could be drawn. As there are not yet validated predictive biomarkers of response to dasatinib ${ }^{25}$, assessment of AR, p-Src and p-HER3 in future studies of dasatinib in ER + breast cancer would be of interest.

CDK 4/6 inhibitor therapy in combination with endocrine therapy is now the accepted standard of care for first-line treatment of ER + HER2- MBC due to substantially improved median PFS times of over 2 years compared to endocrine therapy alone. Src enables the emergence of acquired resistance to $E R$ inhibition by forming a signaling complex between ER, EGFR, and Src. ${ }^{20}$ Because Src inhibition may retard acquired resistance to ER blockade, and because dasatinib is well tolerated over several years and the CBR of dasatinib plus letrozole is $71 \%$, this agent could be a candidate "third leg" of a triplet combination with CDK $4 / 6$ and ER inhibitors. Recent preclinical studies have demonstrated interactions between CDKs and Src in cell cycle regulation, ${ }^{28,29}$ and support investigating the addition of dasatinib to CDK $4 / 6$ and ER inhibitors in breast cancer models.

The results of this non-comparative phase-II trial show a median PFS of 20.1 months with the Src inhibitor dasatinib plus letrozole in patients who had not previously received an Al for metastatic disease. The primary endpoint of CBR was $71 \%$ with letrozole plus dasatinib versus the projected CBR of the combination of $56 \%$. The combination of dasatinib with letrozole was generally well tolerated, with a low rate of serious toxicities and some patients remaining on dasatinib longer than $2-3$ years. Preliminary findings of improved BMD in some patients on dasatinib plus letrozole, and of $A R$ and HER family expression in the primary breast cancers of patients who did not benefit from dasatinib plus letrozole could warrant further investigation.

\section{METHODS}

Patient population

Eligible patients were postmenopausal women $\geq 18$ years of age with histologically confirmed ER-positive (defined as $>10 \%$ by immunohistochemistry), HER2-negative, unresectable, locally recurrent or MBC. Patients may have had either measurable or non-measurable (evaluable-only) disease, and could have received $0-1$ prior chemotherapy regimens for MBC. Prior (neo)adjuvant chemotherapy was allowed. Prior adjuvant aromatase inhibitor (Al) was allowed if it had been stopped $>1$ year prior to study entry, but no prior Al therapy was permitted for MBC. Prior tamoxifen was allowed in either the adjuvant or metastatic settings. Intravenous (IV) bisphosphonates were not allowed during the first 8 weeks of study treatment. Other eligibility criteria included Eastern Cooperative Oncology Group (ECOG) performance status of 0-1; adequate hematologic, cardiac, renal, and liver, function. Patients with symptomatic central nervous system (CNS) metastases, or CNS metastases requiring steroid treatment or local therapy were not eligible. Patients with pleural or pericardial effusions of any grade were not eligible. The US Oncology Institutional Review Board (IRB) reviewed and approved the protocol. All participating patients signed the IRB-approved informed consent form.

\section{Study design}

This was a multicenter, phase-ll, randomized, non-comparative study (NCT00696072). Eligible patients were initially stratified by disease-free interval (DFI) from initial breast cancer diagnosis to first locally recurrent or MBC ( $\leq 2$ years versus $>2$ years), and by prior tamoxifen for metastatic disease (yes/no). Patients were then randomized 1:1 to receive letrozole ( $2.5 \mathrm{mg}$ orally daily) alone or with dasatinib ( $100 \mathrm{mg}$ orally daily). Cycle length was defined as 28 days. Patients with disease progression on letrozole alone had the option of crossing over to receive dasatinib plus continued letrozole. Radiologic assessments to follow known disease were performed every 8 weeks, and bone scans were performed on all patients every 16 weeks (more frequently if disease progression suspected). Bone mineral density (BMD) assessment of lumbar spine and total hip by DXA scan was performed within 4 weeks prior to registration, and at the end of cycle 6. Bisphosphonate treatment was allowed after the first 8 weeks of study treatment at the discretion of the investigator.

\section{Study objectives}

The primary objective of this study was to determine clinical benefit rate (CBR; complete response + partial response + stable disease $\geq 6$ months) with letrozole plus dasatinib or letrozole monotherapy. Secondary objectives included overall response rates (ORR), median progressionfree survival (PFS), 6- and 12-month PFS rates, overall survival (OS), changes in BMD from baseline to on-study assessment, safety, and evaluation of potential biomarkers to predict sensitivity and/or resistance to dasatinib.

\section{Safety assessment}

Safety was assessed throughout the study. Adverse events (AEs) were graded and reported according to the National Cancer Institute Common Terminology Criteria for Adverse Events (version 3.0).

\section{Exploratory biomarker analysis}

Formalin fixed paraffin embedded ((FFPE) primary breast cancer tissue was sent to a Clinical Laboratory Improvement Amendments (CLIA)-certified laboratory (Theranostics Health; Rockville, MD) for analysis by reverse phase protein array (RPPA). Sample protein lysates obtained from microdissected FFPE breast cancer tissues were printed onto slides. Samples were considered "acceptable" for analysis if the protein concentration of the undiluted sample following tumor enrichment and lysate preparation was $\geq 0.1 \mathrm{mg} / \mathrm{mL}$. In addition, samples were eliminated prior to processing if the tumor content (percentage and total number of tumor cells within a section) of a particular sample was insufficient for processing. Immunostaining was carried out with 20 antibodies directed against HER pathway proteins, as well as AKT, S6 Ribosomal Protein, 4EBP1, ERK 1/2, IGFR1, MET, Src, MEK 1/2, STAT3, JAK-2, mTOR, Androgen Receptor (AR and phospho-AR) and the downstream target of Src, paxillin (see Supplemental Table 1 for specific antibody epitopes). Each protein biomarker was scored against the level of expression or activation (phosphorylation) across a population of breast cancers. Patient scores for each protein are based upon the number of standard deviations (SDs) from the population mean.

\section{Statistical analysis}

Planned accrual was 60 patients per arm, to ensure at least 55 efficacyevaluable patients per arm, for a total target accrual of 120 patients. This sample size provided $80 \%$ power to detect an absolute improvement in CBR from $39 \%$ with single agent letrozole to $56 \%$ with letrozole plus dasatinib, using a binomial test with 1-sided alpha level of 5\%, allowing for a $10 \%$ rate of early drop-outs. The CBR of $39 \%$ with letrozole alone was extrapolated from the original first-line trial of letrozole where the CBR was $49 \% ;^{15}$ we estimated a lower CBR in this trial assuming some adjuvant $\mathrm{Al}$ exposure in a proportion of patients. Because this was a randomized non-comparative study using a concurrent control arm, no statistical comparisons between the 2 arms are reported. The intent-totreat (ITT) population included all patients registered on the study, and was used to describe the baseline demographics and disease characteristics, and for the PFS analyses. The evaluable population included all treated patients who met all inclusion and exclusion criteria and who received at least 1 dose of study drug, and was used for analysis of CBR and ORR. The safety population included all patients (eligible and ineligible) who received at least 1 dose of study drug, and was used in analyzing all safety parameters.

CBR was defined as the percentage of patients with complete response $(\mathrm{CR}), \mathrm{PR}$, and stable disease (SD) $\geq 6$ months. ORR was defined as the percentage of $\mathrm{CRs}$ and PRs. The $95 \%$ confidence intervals $(\mathrm{Cl})$ were estimated for both ORR and CBR assuming binomial distribution. A Cochran-Mantel-Haenszel test was used to estimate the differences between ORRs and CBRs in the two arms after accounting for stratification 
factors. PFS was defined as time from date of registration to date of (first) progression or date of death. Patients without disease progression were censored at the last contact date. Kaplan-Meier methods were used to estimate PFS rates and median PFS using the intent-to-treat (ITT) population. Incidence and type of AEs were tabulated and summarized using descriptive statistics.

Student's $t$-test or Mann-Whitney $U$ test and Spearman correlation coefficient $(\rho)$ were used to assess biomarker association with PFS $\leq 6$ months vs $>6$ months by treatment arm (unpaired 2 tails, $p$ value). $P$ values for the biomarker comparisons are exploratory, and have not been adjusted for multiplicity of comparisons.

\section{Reporting summary}

Further information on research design is available in the Nature Research Reporting Summary linked to this article.

\section{DATA AVAILABILITY}

The data generated and analyzed during this study are described in the following data record: https://doi.org/10.6084/m9.figshare.9792056. ${ }^{30}$ The datasets supporting the figures, tables and supplementary figures and tables in this published article are not publicly available in accordance with the policy of US Oncology Research, LLC, and the informed consents signed by the patients. Data supporting the figures, tables, and supplementary figures, can be accessed from the Vice President of US Oncology Research, Dr. Sandy Smith, on request, as described in the data record above. The data supporting the supplementary tables in the published article are not available from the US Oncology Research as these data were provided by the Clinical Laboratory Improvement Amendments (CLIA)-certified laboratory (Theranostics Health; Rockville, MD) that performed the experiments. ${ }^{30}$

Received: 21 January 2019; Accepted: 20 September 2019; Published online: 28 October 2019

\section{REFERENCES}

1. Hiscox, S., Morgan, L., Green, T. \& Nicholson, R. I. Src as a therapeutic target in anti-hormone/ anti-growth factor-resistant breast cancer. Endocr. Relat. Cancer 13, S53-S59 (2006).

2. Zhang, X. H. et al. Latent bone metastasis in breast cancer tied to Src-dependent survival signals. Cancer Cell 16, 67-78 (2009).

3. Larsen, S. L. et al. SRC drives growth of antiestrogen resistant breast cancer cell lines and is a marker for reduced benefit of tamoxifen treatment. PLOS ONE 10, e0118346 (2015).

4. Varricchio, L. et al. Inhibition of estradiol receptor/Src association and cell growth by an estradiol receptor alpha tyrosine-phosphorylated peptide. Mol. Cancer Res 5, 1213-1221 (2007).

5. Vallabhaneni, S. et al. Significance of ER-Src axis in hormonal therapy resistance. Breast Cancer Res Treat. 130, 377-385 (2011).

6. Renoir, J. M., Marsaud, V. \& Lazennec, G. Estrogen receptor signaling as a target for novel breast cancer therapeutics. Biochem Pharm. 85, 449-465 (2013).

7. Chu, I. et al. Src promotes estrogen-dependent estrogen receptor alpha proteolysis in human breast cancer. J. Clin. Invest 117, 2205-2215 (2007).

8. Chen, Y. et al. Combined Src and aromatase inhibition impairs human breast cancer growth in vivo and bypass pathways are activated in AZD0530-resistant tumors. Clin. Cancer Res 15, 3396-3405 (2009).

9. Campone, M. et al. Phase II study of single-agent bosutinib, a Src/Abl tyrosine kinase inhibitor, in patients with locally advanced or metastatic breast cancer pretreated with chemotherapy. Ann. Oncol. 23, 610-617 (2012).

10. Soriano, P., Montgomery, C., Geske, R. \& Bradley, A. Targeted disruption of the csrc proto-oncogene leads to osteopetrosis in mice. Cell 64, 693-702 (1991).

11. Hochhaus, A. \& Kantarjian, H. The development of dasatinib as a treatment for chronic myeloid leukemia (CML): from initial studies to application in newly diagnosed patients. J. Cancer Res Clin. Oncol. 139, 1971-1984 (2013).

12. Mayer, E. L. et al. A phase 2 trial of dasatinib in patients with advanced HER2positive and/or hormone receptor-positive breast cancer. Clin. Cancer Res 17, 6897-6904 (2011).

13. Garcia-Gomez, A. et al. Dasatinib as a bone-modifying agent: anabolic and antiresorptive effects. PLOS ONE 7, e34914 (2012).

14. Vandyke, K. et al. The tyrosine kinase inhibitor dasatinib dysregulates bone remodeling through inhibition of osteoclasts in vivo. J. Bone Min. Res 25, 1759-1770 (2010)
15. Mouridsen, H. et al. Superior efficacy of letrozole versus tamoxifen as first-line therapy for postmenopausal women with advanced breast cancer: results of a phase III study of the International Letrozole Breast Cancer Group. J. Clin. Oncol. 19, 2596-2606 (2001).

16. Finn, R. S. et al. Palbociclib and letrozole in advanced breast cancer. N. Engl. J. Med 375, 1925-1936 (2016).

17. Hortobagyi, G. N. et al. Ribociclib as first-line therapy for HR-positive advanced breast cancer. N. Engl. J. Med 375, 1738-1748 (2016).

18. Llombart, A. et al. Randomized phase II study of dasatinib vs placebo in addition to exemestane in advanced ER/PR-positive breast cancer [BMS CA180-261 study]. Cancer Res. 71, (24 Suppl):Abstract nr PD01-02 (2011).

19. Wright, G. L., et al. Fulvestrant with or without dasatinib in postmenopausal patients with hormone receptor-positive metastatic breast cancer previously treated with an aromatase inhibitor. Cancer Res 71, (24 Suppl):Abstract nr PD0101 (2011).

20. Hitosugi, T. et al. Epidermal growth factor directs sex-specific steroid signaling through Src activation. J. Biol. Chem. 282, 10697-10706 (2007).

21. Moy, B. et al. Bosutinib in combination with the aromatase inhibitor letrozole: a phase II trial in postmenopausal women evaluating first-line endocrine therapy in locally advanced or metastatic hormone receptor-positive/HER2-negative breast cancer. Oncologist 19, 348-349 (2014).

22. Moy, B. et al. Bosutinib in combination with the aromatase inhibitor exemestane: a phase II trial in postmenopausal women with previously treated locally advanced or metastatic hormone receptor-positive/HER2-negative breast cancer. Oncologist 19, 346-347 (2014).

23. Onishi, T., Hayashi, N., Theriault, R. L., Hortobagyi, G. N. \& Ueno, N. T. Future directions of bone-targeted therapy for metastatic breast cancer. Nat. Rev. Clin. Oncol. 7, 641-651 (2010).

24. Araujo, J. \& Logothetis, C. Targeting Src signaling in metastatic bone disease. Int J. Cancer 124, 1-6 (2009).

25. Higgins, M. J. et al. A randomized, double-blind phase II trial of exemestane plus MM-121 (a monoclonal antibody targeting ErbB3) or placebo in postmenopausal women with locally advanced or metastatic ER+/PR+, HER2-negative breast cancer. J. Clin. Oncol. 32, 5s (suppl; abstr 587) (2014).

26. De Amicis, F. et al. Androgen receptor overexpression induces tamoxifen resistance in human breast cancer cells. Breast Cancer Res. Treat. 121, 1-11 (2010).

27. Moulder, S. et al. Development of candidate genomic markers to select breast cancer patients for dasatinib therapy. Mol. Cancer Ther. 9, 1120-1127 (2010).

28. Tsytlonok, M. et al. Dynamic anticipation by Cdk2/Cyclin A-bound p27 mediates signal integration in cell cycle regulation. Nat. Commun. 10, 1676 (2019).

29. Horiuchim, M. et al. The tyrosine kinase v-Src causes mitotic slippage by phosphorylating an inhibitory tyrosine residue of Cdk1. J. Biol. Chem. 293, 15524-15537 (2018).

30. Paul, D. et al. Metadata supporting data files of the related article: randomized phase-II evaluation of letrozole plus dasatinib in hormone receptor positive metastatic breast cancer patients. figshare. https://doi.org/10.6084/m9. figshare.9792056 (2019)

\section{ACKNOWLEDGEMENTS}

The authors thank all of the patients, US Oncology Research physicians and clinical research coordinators who participated in this study; and Susan R. Peck of McKesson Specialty Health for editorial assistance. Study supported by a research grant from Bristol-Myers Squibb, as study CA180185.

\section{AUTHOR CONTRIBUTIONS}

D.P., J.O'.S., Y.W., L.A. and L.S. contributed in the design of the study and the writing of the protocol. Y.W. extracted the data and performed the analysis. All authors read, reviewed, and approved the protocol and the final manuscript. The tables and figures in this manuscript are the original ones.

\section{COMPETING INTERESTS}

Drs. Paul, Vukelja, Holmes, Blum, McIntyre, Lindquist, Sanchez, Goldschmidt, Wang and Asmar reported no conflicts of interest. Dr. Osborne participated in advisory boards for Agendia and Guardant. Dr. Goldschmidt has received honoraria and speaker's fees from Amgen, Eli Lilly, Bristol-Myers Squibb, and Genentech. Dr. O'Shaughnessy has been a consultant for Bristol-Myers Squibb, Agendia, Lilly, Novartis, Pfizer, Genentech, Roche, Merck, Odonate, Arch Oncology, CytomX, Genomic Health, Puma, Synthon, AstraZeneca, Abbvie, Nektar, Halozyme, Eisai, Celgene, Seattle Genetics, Amgen, Jounce, Pharmamar, Grail, 2X Oncology, Myriad, Biothera, Tempus, Oncomed, Carrick, Tocagen, Dompe, Kyoma Kirin, Loxo Oncology, 
Hengrui, Almac, Celldex, Immunomedics. Dr. Strauss is employed by and owns stock in Bristol-Myers Squibb.

\section{ADDITIONAL INFORMATION}

Supplementary information is available for this paper at https://doi.org/10.1038/ s41523-019-0132-8.

Correspondence and requests for materials should be addressed to J.O.

Reprints and permission information is available at http://www.nature.com/ reprints

Publisher's note Springer Nature remains neutral with regard to jurisdictional claims in published maps and institutional affiliations.
Open Access This article is licensed under a Creative Commons Attribution 4.0 International License, which permits use, sharing, adaptation, distribution and reproduction in any medium or format, as long as you give appropriate credit to the original author(s) and the source, provide a link to the Creative Commons license, and indicate if changes were made. The images or other third party material in this article are included in the article's Creative Commons license, unless indicated otherwise in a credit line to the material. If material is not included in the article's Creative Commons license and your intended use is not permitted by statutory regulation or exceeds the permitted use, you will need to obtain permission directly from the copyright holder. To view a copy of this license, visit http://creativecommons. org/licenses/by/4.0/.

(c) The Author(s) 2019 